\title{
AVALIAÇÃO DO DESEMPENHO DE SURFACTANTES PARA A SOLUBILIZAÇÃO DE FASES LÍQUIDAS NÃO AQUOSAS EM MEIO AQUOSO
}

\author{
Elizabeth Fátima de Souza*, Mariana Rodrigues Peres e Samara Boaventura de Moraes \\ Faculdade de Química, Centro de Ciências Exatas, Ambientais e de Tecnologias, Pontifícia Universidade Católica de Campinas, \\ CP 317, 13012-970 Campinas - SP, Brasil
}

Recebido em 10/2/09; aceito em 7/10/09; publicado na web em 11/3/10

\begin{abstract}
EVALUATING SURFACTANT PERFORMANCE AS SOLUBILIZER OF NON-AQUEOUS PHASE LIQUIDS WITHIN AQUEOUS MEDIA. The presence of non-aqueous phase liquids (NAPLs) in the subsurface is a threat to public health as well as a serious environmental issue. NAPLs may remain adsorbed or form lenses floating on aquifers causing long-term contaminations. Surfactants may increase NAPLs solubility, enhancing the pump-and-treatment performance. Size, shape, hydration and ionization degree of the micelles define the affinity and the space available for the solubilization of a particular contaminating agent. The tests carried out at laboratory scale, taking into account the NAPL to be removed and the medium characteristics were useful to select surfactants and evaluate their efficiency as NAPLs solubilizers.
\end{abstract}

Keywords: surfactants; NAPLs; remediation.

\section{INTRODUÇÃO}

A poluição das águas superficiais de rios e lagos já é reconhecida como um problema ambiental há muito tempo. Porém, por não serem imediatamente visíveis, as consequências do descarte indevido de resíduos no solo e a contaminação das águas subterrâneas foram praticamente negligenciadas até a década de $70 .{ }^{1} \mathrm{Na}$ verdade, este é um problema ambiental de grandes proporções, principalmente em países industrializados e em desenvolvimento. Foram identificadas dezenas de milhares de áreas contaminadas de solos no Brasil, Estados Unidos, Canadá, Alemanha, Holanda, França, Rússia e outros países, que estão em ou necessitam de tratamento. ${ }^{2}$ No estado de São Paulo, até novembro de 2008, a CETESB havia identificado 2.514 áreas contaminadas, com cerca de $85 \%$ destas contendo hidrocarbonetos alifáticos e aromáticos (benzeno, tolueno e xilenos, BTX), hidrocarbonetos poliaromáticos (PAH), hidrocarbonetos clorados e metais pesados. ${ }^{3}$ Portanto, entre os tipos mais comuns de contaminação de solos e águas subterrâneas estão os que provem de vazamentos de combustíveis e solventes orgânicos, as chamadas fases não aquosas líquidas (Non-Aqueous Phase Liquids, NAPLs). ${ }^{4}$ A contaminação de aquíferos por NAPLs é preocupante, principalmente quando se considera a disponibilidade dos recursos hídricos, a mobilidade das águas subterrâneas e a possibilidade de deslocamento de plumas de contaminantes para fora do domínio físico da área originalmente contaminada. ${ }^{5}$

Fases líquidas não aquosas (NAPLs) são formadas por compostos orgânicos líquidos que, em função das diferenças de propriedades físicas e químicas, existem como fases distintas e imiscíveis em contato com a água. As fases líquidas não aquosas são usualmente classificadas como fases líquidas não aquosas leves (Light Non-Aqueous Phase Liquids, LNAPLs), com densidades menores ou como fases líquidas não aquosas densas (Dense Non-Aqueous Phase Liquids, DNAPLs), com densidades maiores do que a da água. Benzeno, tolueno e xilenos e outros hidrocarbonetos derivados de petróleo formam LNAPLs. ${ }^{4,6}$ Percloroetileno, clorofórmio, tetracloreto de carbono e outros hidrocarbonetos clorados alifáticos, extensivamente utilizados nas indústrias eletrônica, química e metal-mecânica e nas lavanderias a seco, formam DNAPLs. ${ }^{7}$

\footnotetext{
*e-mail: souzaef@puc-campinas.edu.br
}

Quando ocorre um grande vazamento de NAPLs no solo, elas migram através do solo como uma fase separada, sob a influência da gravidade, deixando gotas residuais na zona não-saturada. Depois de chegar ao lençol freático, as LNAPLs formam lentes que flutuam sobre a fase aquosa, enquanto as DNAPLs se depositam sobre os estratos impermeáveis do subsolo (Figura 1). ${ }^{4,8}$ No subsolo, as LNAPLs apresentaram maior tendência de migração horizontal, concomitante ao movimento da água subterrânea, enquanto as DNAPLs apresentaram uma tendência mais acentuada de migração no sentido vertical. ${ }^{9}$ Ao mesmo tempo, os constituintes das NAPLs podem vir a sofrer partição, ou mover-se de fase a outra, dependendo pressão de vapor da substância e sua solubilidade em água e das condições ambientais. ${ }^{4,8}$

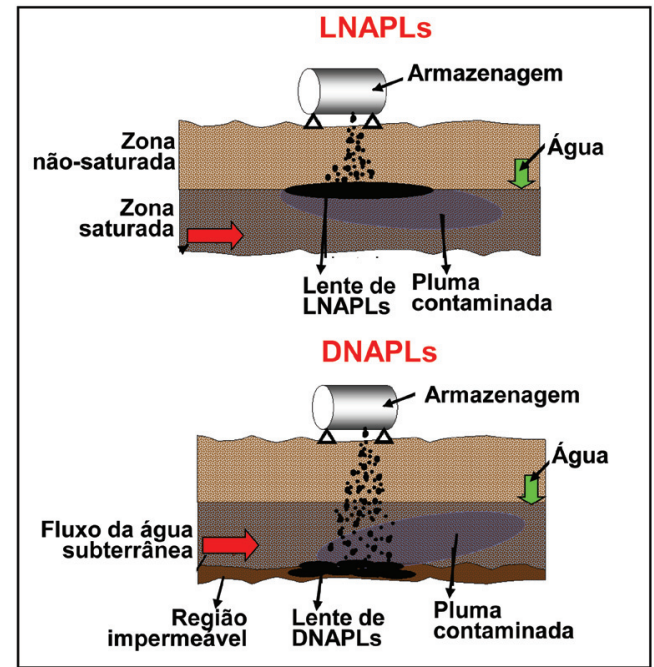

Figura 1. Esquema representando a contaminação do subsolo e de aquíferos causada por vazamentos de (a) LNAPLs e (b) DNAPLs. Adaptada da ref. 7

A extensão dos problemas de contaminação de solos por NAPLs e incertezas sobre a sua migração e distribuição fazem com que seja necessário o desenvolvimento de estratégias consistentemente eficientes para a remediação das áreas impactadas. ${ }^{4,5,10} \mathrm{~A}$ necessidade de conter as contaminações de aquíferos já identificadas em seus 
locais de origem levou à aplicação, em larga escala, de sistemas de bombeamento e tratamento. Esta técnica consiste na extração das águas contaminadas do subsolo para o controle das fontes e remoção de massa dos contaminantes e no tratamento ex-situ dos efluentes, de modo a satisfazer os critérios ambientais. Porém, depois de mais de uma década de utilização, verificou-se que a utilização do bombeamento e tratamento como única forma de remediação não é suficiente, na maioria dos casos, para promover a reabilitação da área contaminada de forma rápida e com custos aceitáveis. Ainda assim, os sistemas de bombeamento e tratamento de águas subterrâneas podem se constituir em uma parte importante do programa de remediação de solos e aquíferos contaminados, controlando a migração de uma pluma de contaminantes para regiões mais afastadas do aquífero e permitindo a remoção de fontes secundárias da contaminação. ${ }^{11}$

A adição de surfactantes durante a lavagem de solo pode reduzir a tensão superficial entre os contaminantes e a água, promovendo a mobilização da fase orgânica residual e imiscível a ser extraída. ${ }^{5}$ A mobilização intensificada por surfactante utiliza baixas tensões interfaciais para reduzir significativamente as forças capilares que prendem as NAPLs, permitindo assim que a fase orgânica seja extraída facilmente como uma fase separada. ${ }^{12}$ Já a solubilização intensificada por surfactantes aumenta significativamente a concentração aquosa da NAPL via partição micelar, reduzindo assim o número de volumes de poros de água que deve ser bombeado do subsolo para extrair as NAPLs. A solubilização de fases orgânicas por soluções de surfactantes é induzida pela sua capacidade de formação de micelas acima da concentração micelar crítica (CMC). O núcleo hidrofóbico das micelas pode armazenar e transportar moléculas orgânicas, aumentando a solubilidade aparente dos compostos orgânicos na fase aquosa em algumas ordens de magnitude e proporcionando maior eficiência ao bombeamento e tratamento. ${ }^{12,13} \mathrm{~A}$ remediação de aquíferos intensificada por surfactantes (Surfactant-Enhanced Aquifer Remediation - SEAR) é, portanto, uma das alternativas existentes para aumentar a efetividade do bombeamento e tratamento, através da mobilização e/ou solubilização das NAPLs. ${ }^{5,13}$

Tecnologias de remediação à base de surfactantes têm sido o foco de diversas pesquisas e foram, recentemente, aplicadas em estudos de campo para a avaliação da aplicabilidade em larga escala. Os principais fatores que devem ser considerados para a seleção de um surfactante para remediação de solos contaminados são: eficiência, custos, biodegradabilidade, toxicidade para os seres vivos e possibilidade de reciclagem. ${ }^{5,13,14}$ Desta forma, o objetivo principal deste trabalho foi testar e identificar em escala de laboratório os fatores que influenciam o desempenho de soluções de surfactantes para a solubilização de NAPLs.

\section{PARTE EXPERIMENTAL}

\section{Material}

Os solventes decano $\left(\mathrm{C}_{10} \mathrm{H}_{22}\right.$, Aldrich $)$ e tolueno $\left(\mathrm{C}_{6} \mathrm{H}_{5} \mathrm{CH}_{3}, \mathrm{Al}\right.$ drich), tetracloreto de carbono $\left(\mathrm{CCl}_{4}, \mathrm{Aldrich}\right)$, clorofórmio $\left(\mathrm{CHCl}_{3}\right.$, Aldrich) e 1,2-dicloroetano $\left(\mathrm{C}_{2} \mathrm{H}_{4} \mathrm{Cl}_{2}, \mathrm{Aldrich}\right)$ foram escolhidos para os ensaios preliminares de identificação do potencial dos surfactantes testados para a solubilização de fases líquidas não aquosas.

Como a maioria dos solventes orgânicos é incolor, muitos pesquisadores utilizaram, em escala de laboratório, corantes hidrofóbicos, para distinguir visualmente as NAPLs da fase aquosa ou para identificar a solubilização das NAPLs por soluções de surfactantes através do coramento da fase aquosa. ${ }^{15}$ Os corantes Oil Blue $\mathrm{N}$ (1,4-di(pentilamino)antraquinona, $\mathrm{C}_{24} \mathrm{H}_{30} \mathrm{~N}_{2} \mathrm{O}_{2}$, Aldrich) e Oil Red $\mathrm{O}$ (1-((4-(dimetilfenil)azo)dimetilfenil)azo)-2-naftalenol, $\mathrm{C}_{26} \mathrm{H}_{24} \mathrm{~N}_{4} \mathrm{O}$, Aldrich) são solúveis nos solventes orgânicos e insolúveis em água, possuindo as características necessárias para serem utilizados nos testes de solubilização de NAPLs.

Foram testados os surfactantes não iônicos mono-oleato de sorbitan (Tween 80), monoestearato de sorbitan (Tween 60), monopalmitato de sorbitan (Tween 40) e octilfenol (Triton X-100) etoxilados, os números indicando os diferentes graus de etoxilação de cada um. O surfactante cationnico testado foi o brometo de hexadeciltrimetilamônio (CTAB) e o aniônico foi o laurilsulfato de sódio (SDS). As Tabelas 1 e 2 apresentam algumas das propriedades dos compostos orgânicos e surfactantes utilizados neste trabalho.

As soluções de surfactantes e de solventes orgânicos corados foram preparadas, com técnicas usuais de volumetria, a partir dos reagentes puros tal como recebidos (Aldrich, Sigma ou Vetec) e água deionizada (Milli-Q, resistividade de $\sim 18 \mathrm{M} \Omega / \mathrm{cm}$ ) nas condições desejadas.

Tabela 1. Algumas propriedades físico-químicas da água e de compostos orgânicos representativos de NAPLs nas condições de temperatura e pressão ambiente. Adaptada da ref. 16

\begin{tabular}{|c|c|c|c|c|c|c|}
\hline $\begin{array}{l}\text { Propriedade } \\
\text { Composto }\end{array}$ & $\begin{array}{c}\text { Tensão superficial } \\
\left(\text { dinas } \mathrm{cm}^{-1}\right)\end{array}$ & $\begin{array}{l}\text { Densidade } \\
\left(\mathrm{g} \mathrm{mL}^{-1}\right)\end{array}$ & $\begin{array}{l}\text { Viscosidade } \\
(\mathrm{cP})\end{array}$ & $\begin{array}{l}\text { Solubilidade em água } \\
(\mathrm{ppm})\end{array}$ & $\begin{array}{l}\text { Pressão de vapor } \\
\quad(\mathrm{mm} \mathrm{Hg})\end{array}$ & $\begin{array}{l}\text { Volume molar } \\
\left(\mathrm{cm}^{3} \mathrm{~mol}^{-1}\right)\end{array}$ \\
\hline Água & 72,2 & 0,999 & 1,02 & -- & -- & 18,0 \\
\hline Tolueno & 55,1 & 0,868 & 0,59 & 500 & 22 & 105,7 \\
\hline Decano & 24,3 & 0,728 & 0,92 & 0,052 & 1,3 & 193,6 \\
\hline Tetracloreto de carbono & 35,2 & 1,697 & 0,95 & 800 & 90 & 90,6 \\
\hline Clorofórmio & 28,9 & 1,500 & 0,50 & 8000 & 160 & 79,5 \\
\hline Dicloroetano & 25,0 & 1,173 & 1,5 & 8690 & 64 & 84,3 \\
\hline
\end{tabular}

Tabela 2. Propriedades selecionadas dos surfactantes utilizados ${ }^{7,17}$

\begin{tabular}{|c|c|c|c|c|c|c|}
\hline Surfactante & Fórmula molecular & $\begin{array}{l}\text { Massa molar } \\
\quad\left(\mathrm{g} \mathrm{mol}^{-1}\right)\end{array}$ & $\begin{array}{c}\mathrm{CMC} \\
\left(\mathrm{mol} \mathrm{L}^{-1}\right)\end{array}$ & HLB & $\begin{array}{l}\text { Número médio } \\
\text { de agregação }\end{array}$ & $\begin{array}{c}\text { Grau } \\
\text { de ionização }\end{array}$ \\
\hline CTAB & $\mathrm{CH}_{3}\left(\mathrm{CH}_{2}\right)_{15} \mathrm{~N}\left(\mathrm{CH}_{3}\right)_{3} \mathrm{Br}$ & 364,5 & $9,2 \times 10^{-4}$ & 10 & 95 & $0,2-0,1$ \\
\hline SDS & $\mathrm{CH}_{3}\left(\mathrm{CH}_{2}\right)_{11} \mathrm{SO}_{4} \mathrm{Na}$ & 288,4 & $8,2 \times 10^{-3}$ & -- & 50 & 0,3 \\
\hline Triton X100 & $\mathrm{C}_{8} \mathrm{H}_{17} \mathrm{C}_{6} \mathrm{H}_{4}\left(\mathrm{C}_{2} \mathrm{H}_{4} \mathrm{O}\right)_{9} \mathrm{OH}$ & 625 & $2,2 \times 10^{-4}$ & 13,5 & 140 & -- \\
\hline Tween 40 & $\mathrm{C}_{62} \mathrm{H}_{122} \mathrm{O}_{26}$ & 1284 & $2,7 \times 10^{-5}$ & 15,6 & $90-95$ & -- \\
\hline Tween 60 & $\mathrm{C}_{64} \mathrm{H}_{126} \mathrm{O}_{26}$ & 1310 & $1,5 \times 10^{-5}$ & 15 & 100 & -- \\
\hline Tween 80 & $\mathrm{C}_{64} \mathrm{H}_{124} \mathrm{O}_{26}$ & 1310 & $1,2 \times 10^{-5}$ & 15 & 110 & -- \\
\hline
\end{tabular}


Identificação do potencial dos surfactantes para solubilização de NAPLs

A avaliação inicial do potencial de solubilização um determinado surfactante foi realizada de acordo com um procedimento originalmente proposto por Zhou e Rhue ${ }^{18}$ e modificado por Imazaki et al.: ${ }^{19} 25 \mathrm{~mL}$ da solução de surfactante $(2,5 \% \mathrm{~m} / \mathrm{v})$ foram transferidos para um frasco de $125 \mathrm{~mL}$, ao qual foram adicionados $0,5 \mathrm{~mL}$ da NAPL desejada previamente corada com $0,001 \mathrm{~g} / \mathrm{mL}$ do corante desejado. Cada frasco foi agitado várias vezes durante um período de $24 \mathrm{~h}$ e, a seguir, permaneceu em repouso por $24 \mathrm{~h}$. A avaliação do desempenho do surfactante foi realizada pela inspeção visual da intensidade de cor da fase aquosa e da quantidade de NAPL não solubilizada, presente como uma segunda fase no sistema. A seguir, foram obtidos os espectros de absorção de luz na região UV-visível (190 a $1090 \mathrm{~nm}$ ) das fases aquosas, utilizando-se um espectrofotômetro UV/VIS HewlettPackard $8351 .{ }^{19} \mathrm{~A}$ intensidade da solubilização dos contaminantes no interior das micelas das soluções de surfactantes testadas foi classificada a partir do coramento da fase aquosa, utilizando-se os valores das absorbâncias medidas no comprimento de onda característico do corante (Oil Red, $530 \mathrm{~nm}$; Oil Blue, $655 \mathrm{~nm}$ ). As fases aquosas dos testes de solubilização realizados em durezas elevadas foram filtradas através de uma membrana (diâmetro de poro de $0,47 \mu \mathrm{m}$ ) antes da obtenção dos espectros de absorbância, para a remoção de eventuais partículas de carbonato de cálcio dispersas no meio líquido.

\section{Planejamento fatorial para a identificação da influência das condições do meio sobre o desempenho dos surfactantes na solubilização de NAPLs}

Um planejamento fatorial permite a análise simultânea do efeito de cada fator e de suas interações, bem como a identificação dos fatores significativos para a resposta desejada, analisando níveis de cada fator. Assim, para identificação da influência das condições do meio sobre o desempenho dos surfactantes na solubilização de NAPLs foi proposto um planejamento fatorial com três fatores em dois níveis $2^{3}$, em que os parâmetros avaliados foram temperatura, $\mathrm{pH}$ e dureza da fase aquosa com o surfactante dissolvido, de acordo com a Tabela 3. Todos os testes foram realizados em triplicata. Para o cálculo do efeito global apresentado pelas variáveis testadas, foram utilizadas as planilhas eletrônicas para cálculos de planejamentos fatoriais desenvolvidas por Teófilo e Ferreira. ${ }^{20}$

Tabela 3. Condições do planejamento fatorial $2^{3}$ para otimização das condições de solubilização das NAPL coradas por soluções $2,5 \% \mathrm{~m} / \mathrm{v}$ do surfactante desejado

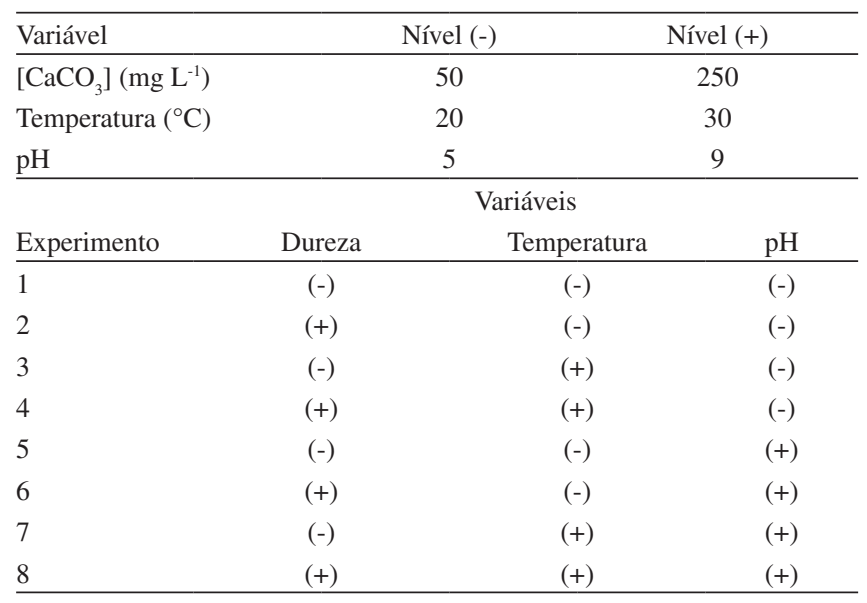

\section{RESULTADOS E DISCUSSÃO}

Influência do corante e da fase orgânica sobre o desempenho dos surfactantes na solubilização de NAPLs

Os primeiros testes foram realizados em temperatura ambiente, sem alteração de $\mathrm{pH}$ ou de dureza da água deionizada utilizada no preparo das soluções. Dos corantes testados, o Oil Blue foi o único que apresentou solubilização total nas LNAPLs testadas (tolueno e n-decano), sendo o escolhido para os testes de desempenho dos surfactantes na solubilização de fases orgânicas leves. Isto decorre, provavelmente, da presença de uma cadeia alifática maior no Oil Blue do que nos outros corantes, o que confere a ele uma maior afinidade com os hidrocarbonetos testados (decano e tolueno). O Oil Red foi bem solubilizado tanto nas LNAPLs, quanto nas DNAPLs testadas (tetracloreto de carbono, clorofórmio e 1,2-dicloroetano).

A Figura 2 apresenta uma comparação entre os espectros UV/ vis obtidos durante a tentativa de solubilização da LNAPL decano corada com Oil Blue por soluções dos surfactantes Tween 40 e CTAB.

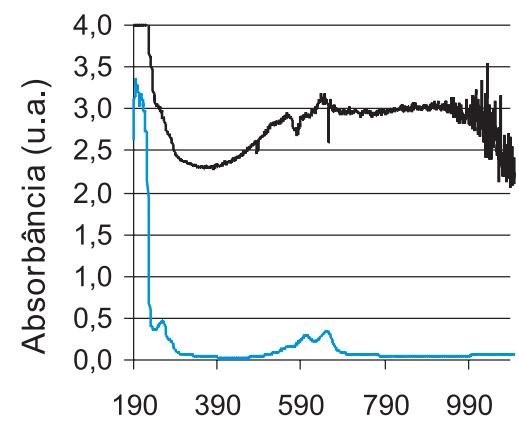

Figura 2. Espectros UV/vis da fase aquosa de soluções de surfactantes 2,5\% $\mathrm{m} / \mathrm{v}$ contaminadas com decano corado com Oil Blue, após 48 h de contato na temperatura de $25^{\circ} \mathrm{C}$

No espectro UV/vis da fase aquosa contendo o surfactante Tween 40 a $25^{\circ} \mathrm{C}$, ocorreu a emulsificação da fase orgânica na aquosa, indicada por um forte espalhamento de luz em toda a região do visível. Um espectro de absorção de luz com valores de absorbâncias extremamente elevados e com flutuações acentuadas em praticamente toda a faixa de comprimentos de onda do ultravioleta e do visível é típico do fenômeno de espalhamento de luz e não do fenômeno de absorção. O espalhamento ocorre na interface água/óleo das gotículas de fase orgânica emulsionada na fase aquosa, em todos os comprimentos de onda. ${ }^{21}$ No caso da fase aquosa com CTAB a $25^{\circ} \mathrm{C}$, ocorreu apenas a absorção de luz nos comprimentos de onda característicos do corante Oil Blue, indicando a solubilização da fase orgânica corada nas micelas do surfactante. Para as DNAPLs também foi observado, em alguns casos, um comportamento similar ao descrito acima para as LNAPLs, isto é, a emulsificação da fase orgânica (Figura 1S, material suplementar). Por este motivo, os resultados considerados nos demais testes foram os obtidos com surfactantes e em condições nas quais ocorreu apenas a solubilização e não a emulsificação do contaminante orgânico na fase aquosa.

O desempenho de um mesmo surfactante no processo de solubilização de DNAPLs é bastante influenciado pelo tipo de corante utilizado na identificação da fase orgânica como mostra a Figura 3.

Como se pode observar, as soluções dos surfactantes testados solubilizaram melhor o organoclorado tetracloreto de carbono quando este foi corado com Oil Red. Porém, os surfactantes Tween 80 e SDS solubilizaram melhor o clorofórmio e os surfactantes Tween 80 , CTAB e SDS solubilizaram melhor o 1,2-dicloroetano na presença de Oil Blue (Figura 2S, material suplementar). Organoclorados 


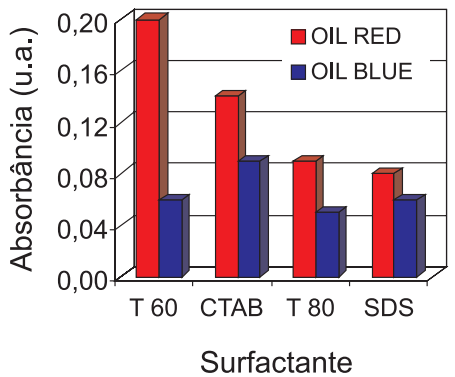

Figura 3. Comparação do desempenho de solubilização de tetracloreto de carbono corado com Oil Red ou Oil Blue por soluções 2,5\% $\mathrm{m} / \mathrm{v}$ dos surfactantes CTAB, SDS, Tween 60 ou Tween 80, após 48 h de contato na temperatura de $25{ }^{\circ} \mathrm{C}$, por medida da absorbância da fase aquosa no comprimento de onda típico do corante

em que as ligações $\mathrm{C}$-Cl não estão simetricamente distribuídas na molécula, como no caso do clorofórmio e o 1,2-dicloroetano, apresentam alguma polaridade e uma maior solubilidade em água (Tabela 1). Esta característica deveria facilitar a aproximação destes compostos da região hidrofílica das micelas em contato com a fase aquosa e, posteriormente, a sua solubilização no interior das micelas, independentemente do fato do surfactante ser catiônico, aniônico ou não iônico. Porém, a presença do corante modifica a tensão interfacial entre a água e o composto organoclorado ${ }^{14} \mathrm{e}$, dependendo do corante utilizado, esta alteração pode ser mais ou menos intensa. Além disto, pode haver uma maior ou menor facilidade de inserção do organoclorado corado dependendo da afinidade entre o próprio corante e as frações apolares dos surfactantes. Poucos estudos da literatura indicam que a capacidade de solubilização dos surfactantes depende do corante utilizado. ${ }^{19,22}$ Portanto, ao utilizarmos os dados da literatura, em que corantes como o Sudan IV, ${ }^{23}$ o Oil $\operatorname{Red}^{24}$ ou o Azul Brilhante $\mathrm{FCF}^{25}$ são usados para a identificação da solubilização de uma mesma fase orgânica, é necessário considerar que os resultados apresentados dependem do corante utilizado e não podem ser diretamente comparados.

A influência do tipo de composto orgânico no desempenho de solubilização das LNAPLs coradas com Oil Blue por soluções de surfactantes pode ser observada na Figura 4.

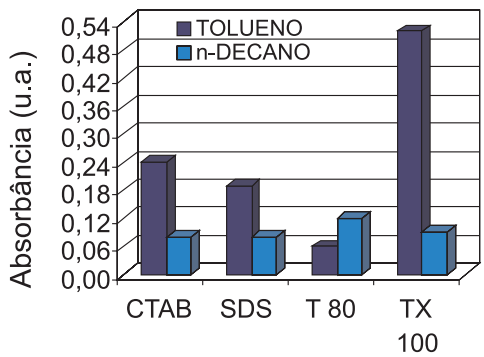

Surfactante

Figura 4. Comparação do desempenho de solubilização de tolueno e de n-decano corados com Oil Blue por soluções 2,5\% $\mathrm{m} / \mathrm{v}$ dos surfactantes CTAB, SDS, Tween 80 ou Triton X-100, após 48 h de contato na temperatura de $25{ }^{\circ} \mathrm{C}$, por medida da absorbância da fase aquosa no comprimento de onda típico do corante

No caso das soluções de surfactantes CTAB, SDS e Triton X-100 a solubilização de tolueno foi maior do que a do decano. Porém, a solução com Triton X-100 apresentou uma leve turvação, indicando o início da emulsificação da fase aquosa. Provavelmente, o tolueno tende a ser mais facilmente solubilizado do que o decano, por apresentar maior taxa de dissolução na interface orgânico/água, uma vez que a captura de moléculas dissolvidas por micelas é rápida. ${ }^{26}$ Além disto, o tolueno é polarizável e pode ser acomodado no interior ou na paliçada das micelas. ${ }^{27}$ Já com a solução do surfactante Tween 80, ocorre o inverso, isto é, a solubilização de decano é maior que a de tolueno, o que pode ser o resultado da presença do corante Oil Blue no sistema. Influência do $\mathrm{pH}$, da dureza e da temperatura da fase aquosa
na solubilização das LNAPLs por soluções de surfactante

Os efeitos isolados dos fatores temperatura, $\mathrm{pH}$ e dureza do meio aquoso na solubilização de tolueno corado com Oil Blue por soluções $2,5 \% \mathrm{~m} / \mathrm{v}$ dos surfactantes CTAB, SDS, TWEEN 80 e Triton X-100 podem ser vistos na Figura 5.
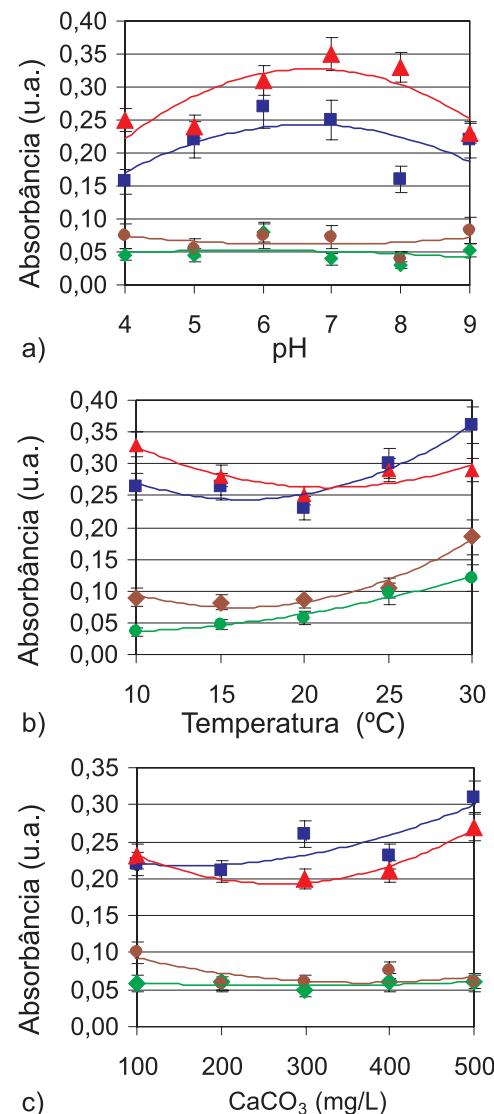

Figura 5. Efeito da temperatura (a), do pH (b) e da dureza (c) na solubilização de tolueno corado com Oil Blue por soluções 2,5\% $\mathrm{m} / \mathrm{v}$ dos surfactantes CTAB, SDS, Tween 80 ou Triton X100, após 48 h de contato na temperatura indicada ou a $25^{\circ} \mathrm{C}$ ( $\mathrm{pH}$ e dureza), por medida da absorbância da fase aquosa no comprimento de onda típico do corante

Na comparação do desempenho de solubilização de tolueno, as soluções de CTAB e Triton X-100 apresentaram melhores desempenhos e maior sensibilidade à variação de $\mathrm{pH}$ do meio. Já o desempenho das soluções de Tween 80 e SDS, com capacidades de solubilização reduzidas, foi pouco influenciado pelo $\mathrm{pH}$ do meio. No caso de micelas de $\mathrm{CTAB}$, em pHs elevados pode ocorrer uma substituição dos contra-íons $\mathrm{Br}$ da camada de Stern por íons $\mathrm{OH}^{-}$, modificando a carga superficial das micelas e dificultando a incorporação dos compostos orgânicos. No caso dos surfactantes não iônicos, o aumento do $\mathrm{pH}$ da fase aquosa tende a diminuir a hidratação das micelas, ${ }^{28}$ o que deveria facilitar a inserção dos contaminantes nos agregados micelares. Porém, surfactantes com um grau de etoxilação elevado, com maiores frações hidrofílicas, como o Tween 80 (20 moléculas de óxido de etileno/mono-oleato de sorbitan), parecem ser menos 
sensíveis às variações de $\mathrm{pH}$ do meio do que surfactantes com menor grau de etoxilação como o Triton X-100 ( 10 moléculas de óxido de etileno/octilfenol). Finalmente, os surfactantes testados com maiores eficiências na solubilização de LNAPLs apresentaram uma faixa ótima de atuação na região de $\mathrm{pH}$ aproximadamente neutro, o que não seria favorável na remediação dos solos brasileiros, visto que a maioria dos solos no Brasil apresenta acidez elevada. ${ }^{29}$

A variação de temperatura tem uma influencia significativa sobre a solubilização do tolueno, que é favorecida com a elevação da temperatura. Como no caso anterior, as soluções dos surfactantes CTAB e Triton X-100 apresentaram os melhores desempenhos de solubilização e as de Tween 80 e SDS foram menos eficientes. Em temperaturas altas, as longas cadeias de poli(oxietileno) das micelas dos surfactantes não iônicos do tipo ésteres etoxilados (Tweens) tornam-se mais desidratadas e hidrofóbicas. ${ }^{30} \mathrm{O}$ mesmo ocorre com surfactantes iônicos como o SDS, ainda que neste caso a situação seja mais complexa devido à existência de repulsão entre os grupos iônicos das cabeças dos surfactantes e ao efeito das moléculas de água na estabilização dos contra-íons junto à superfície das micelas e em solução. ${ }^{31}$ Alguns estudos indicam que o uso de água quente pode ser uma técnica de remediação atraente quando a solubilização do contaminante é significantemente alterada com a temperatura ou se há limitações no bombeamento de fases não aquosas por causa de sua viscosidade. ${ }^{32}$ De acordo com os resultados obtidos, esta técnica poderia ser aplicada no caso da solubilização das LNAPLs testadas.

A variação da dureza da fase aquosa pouco influenciou o desempenho de soluções dos surfactantes Tween 80 e SDS na solubilização de tolueno, mas o desempenho destas soluções foi inferior ao das soluções de CTAB e de Triton X-100. Em durezas elevadas, com o aumento da concentração de sais no meio aquoso, micelas maiores podem ser formadas devido ao aumento da atração intermicelar, ${ }^{33}$ o que, aparentemente, faz com que a incorporação do tolueno nas micelas seja favorecida. Em durezas elevadas, pode ocorrer uma substituição dos contra-íons $\mathrm{Br}^{-}$da camada de Stern de micelas de CTAB por íons $\mathrm{CO}_{3}^{2-}$. A substituição de contra-íons $\mathrm{Na}^{+}$por íons $\mathrm{Ca}^{2+}$ nas micelas de SDS também pode ocorrer, uma vez que a interação entre $\mathrm{Ca}^{2+}$ e a micela é exotérmica, indicando uma situação eletrostática favorável. ${ }^{34}$ Porém, se esta substituição de contra-íons ocorre nas micelas de SDS, não modifica significativamente o desempenho da solução do surfactante.

\section{Influência do pH, da dureza e da temperatura da fase aquosa na solubilização das DNAPLs por soluções de surfactante}

Os efeitos isolados dos fatores temperatura, $\mathrm{pH}$ e dureza do meio aquoso na solubilização de tetracloreto de carbono corado com Oil Red por soluções 2,5\% m/v dos surfactantes CTAB, SDS, Tween 80 e Triton X-100 encontram-se na Figura 3S, material suplementar.

A solução do surfactante Triton X-100 apresentou o melhor desempenho de solubilização do tetracloreto de carbono, com uma leve redução de eficiência em pHs alcalinos. Já a solução de CTAB apresentou seu melhor desempenho nas regiões mais ácida e alcalina, os piores resultados sendo obtidos em $\mathrm{pHs}$ próximos da neutralidade. As soluções dos surfactantes SDS e Tween 80 apresentaram os piores desempenhos de solubilização e menor influência do pH do meio.

$\mathrm{O}$ aumento da temperatura no meio influencia pouco o desempenho de soluções de Triton X-100, CTAB e SDS na solubilização do tetracloreto de carbono, o efeito é significativo apenas para a solução de Tween 80 .

Além disto, é possível observar na Figura 3S, material suplementar, que os surfactantes não iônico (Tween 80) e aniônico (SDS) testados apresentaram um melhor desempenho em durezas elevadas (300 a $500 \mathrm{mg} \mathrm{L}^{-1} \mathrm{de} \mathrm{CaCO}_{3}$ ). Já surfactante catiônico CTAB teve o seu desempenho prejudicado em durezas mais altas, solubilizando melhor a fase orgânica em durezas menores (200 a $300 \mathrm{mg} \mathrm{L}^{-1} \mathrm{de}$ $\mathrm{CaCO}_{3}$ ). No caso do surfactante Triton X-100, os resultados obtidos mostraram a emulsificação da fase orgânica em durezas acima de 100 $\mathrm{mg} / \mathrm{L} \mathrm{de} \mathrm{CaCO}_{3}$ e, portanto, estes não foram incluídos. Porém, como os valores de durezas utilizados neste trabalho ultrapassam as durezas encontradas comumente nas águas subterrâneas brasileiras, ${ }^{28}$ não se espera interferência deste parâmetro no desempenho dos surfactantes em condições de campo.

Finalmente, verificamos que o desempenho de solubilização de LNAPLs e DNAPLs pelas soluções de surfactantes testadas apresenta algumas similaridades: a soluções do surfactante catiônico CTAB e do não iônico Triton X-100 apresentam desempenhos superiores aos das soluções dos surfactantes aniônico SDS e não iônico Tween 80. As influências de $\mathrm{pH}$ e a dureza tendem a ser menores quando se utilizam soluções destes dois últimos, o que provavelmente está associado à baixa capacidade de solubilização dos mesmos. A elevação da temperatura tende a aumentar a eficiência de solubilização, este efeito sendo mais pronunciado na solubilização do tolueno. Já as maiores diferenças que ocorrem na solubilização de tolueno e de tetracloreto de carbono por soluções de CTAB são as faixas ótimas de $\mathrm{pH}$ distintas para a solubilização de tolueno e de tetracloreto de carbono; um efeito da temperatura mais pronunciado no caso do tolueno e o comportamento em durezas elevadas. Estes resultados indicam que as modificações nas micelas do surfactante catiônico, resultantes das modificações nas características da fase aquosa, influem de forma significativa sobre a facilidade de solubilização de uma determinada NAPL.

\section{Aplicação de planejamento fatorial na avaliação do desempenho de surfactantes na solubilização das NAPLs}

A utilização do planejamento fatorial permitiu a análise dos efeitos das variáveis do meio aquoso sobre o desempenho de soluções dos surfactantes na solubilização de NAPLs. A Tabela 4 apresenta os resultados obtidos nos ensaios propostos com o planejamento fatorial para a identificação dos efeitos de $\mathrm{pH}$, dureza e temperatura sobre a solubilização de n-decano e de tolueno corados com Oil Blue por soluções $2,5 \% \mathrm{~m} / \mathrm{v}$ de CTAB, SDS e Tween 40 . Os efeitos de $\mathrm{pH}$, temperatura e dureza sobre o desempenho de solubilização por soluções de Tween 40 não foram calculados nos experimentos com o nível superior de dureza, uma vez que foi detectada a emulsificação da fase orgânica. A Tabela 5 apresenta os resultados obtidos nos ensaios propostos com o planejamento fatorial para a solubilização de tetracloreto de carbono corado com Oil Blue ou Oil Red por soluções $2,5 \% \mathrm{~m} / \mathrm{v}$ de CTAB, SDS e Tween 40.

A Tabela 6 apresenta os efeitos calculados a partir dos resultados das Tabelas 4 e 5, com os valores significativos destacados em negrito. Quando se utilizou o corante Oil Blue para a identificação da fase orgânica, na solubilização de tolueno por soluções de CTAB e de tetracloreto de carbono por soluções de SDS, nenhuma mudança nos níveis dos fatores testados alterou as respostas de forma significativa. Na solubilização de tolueno corado com Oil Blue pela solução do surfactante SDS foi detectado um efeito positivo e significativo quando se passa do nível inferior para o nível superior de $\mathrm{pH}$ (efeito 3). No caso do n-decano corado com Oil Blue este mesmo efeito foi detectado tanto para as soluções de CTAB, como de SDS. Para o tetracloreto de carbono corado com Oil Blue foi detectado um efeito significativo e negativo quando se passa do nível inferior para o superior de dureza (efeito 1) nas soluções de CTAB.

Quando se utilizou o Oil Red para a identificação da fase orgânica, na solubilização do tetracloreto de carbono por soluções de SDS, as 
Tabela 4. Resultados obtidos com o planejamento fatorial $2^{3}$ para estudo das condições do meio sobre a solubilização de n-decano ou de tolueno corado com Oil Blue por soluções $2,5 \% \mathrm{~m} / \mathrm{v}$ de CTAB, SDS e Tween 40

\begin{tabular}{|c|c|c|c|c|c|c|}
\hline \multirow{2}{*}{ Experimento } & \multicolumn{3}{|c|}{ Decano } & \multicolumn{3}{|c|}{ Tolueno } \\
\hline & CTAB & SDS & Tween 40 & CTAB & SDS & Tween 40 \\
\hline 1 & $0,24 \pm 0,04$ & $0,15 \pm 0,05$ & $0,09 \pm 0,03$ & $0,30 \pm 0,09$ & $0,16 \pm 0,03$ & $0,12 \pm 0,05$ \\
\hline 2 & $0,21 \pm 0,02$ & $0,16 \pm 0,01$ & $\ldots$ & $0,34 \pm 0,08$ & $0,13 \pm 0,06$ & $0,17 \pm 0,06$ \\
\hline 3 & $0,24 \pm 0,04$ & $0,16 \pm 0,03$ & $0,15 \pm 0,03$ & $0,30 \pm 0,12$ & $0,15 \pm 0,05$ & $0,17 \pm 0,10$ \\
\hline 4 & $0,25 \pm 0,03$ & $0,16 \pm 0,01$ & -- & $0,35 \pm 0,09$ & $0,14 \pm 0,04$ & $0,18 \pm 0,11$ \\
\hline 5 & $0,27 \pm 0,05$ & $0,17 \pm 0,03$ & $0,07 \pm 0,01$ & $0,37 \pm 0,10$ & $0,25 \pm 0,04$ & $0,25 \pm 0,02$ \\
\hline 6 & $0,29 \pm 0,03$ & $0,19 \pm 0,02$ & -- & $0,39 \pm 0,14$ & $0,25 \pm 0,09$ & $0,27 \pm 0,05$ \\
\hline 7 & $0,23 \pm 0,03$ & $0,18 \pm 0,01$ & $0,15 \pm 0,02$ & $0,38 \pm 0,06$ & $0,21 \pm 0,02$ & $0,28 \pm 0,01$ \\
\hline 8 & $0,30 \pm 0,06$ & $0,21 \pm 0,04$ & -- & $0,41 \pm 0,12$ & $0,27 \pm 0,06$ & -- \\
\hline
\end{tabular}

Resposta: absorbância da fase aquosa após 48 h nas condições desejadas, medida no comprimento de onda do máximo de absorção do corante

Tabela 5. Resultados obtidos com o planejamento fatorial $2^{3}$ para estudo das condições do meio sobre a solubilização de tetracloreto de carbono corado com Oil Blue ou Oil Red por soluções $2,5 \% \mathrm{~m} / \mathrm{v}$ de CTAB, SDS e Tween 40

\begin{tabular}{|c|c|c|c|c|c|c|}
\hline \multirow{2}{*}{ Experimento } & \multicolumn{3}{|c|}{ Oil Blue } & \multicolumn{3}{|c|}{ Oil Red } \\
\hline & CTAB & SDS & Tween 40 & CTAB & SDS & Tween 40 \\
\hline 1 & $0,15 \pm 0,02$ & $0,06 \pm 0,02$ & $0,10 \pm 0,02$ & $0,14 \pm 0,04$ & $0,05 \pm 0,03$ & $0,11 \pm 0,01$ \\
\hline 2 & $0,09 \pm 0,03$ & $0,07 \pm 0,01$ & $0,12 \pm 0,02$ & $0,17 \pm 0,06$ & $0,05 \pm 0,07$ & $0,29 \pm 0,04$ \\
\hline 3 & $0,17 \pm 0,04$ & $0,09 \pm 0,04$ & $0,13 \pm 0,03$ & $0,16 \pm 0,02$ & $0,05 \pm 0,02$ & $0,10 \pm 0,02$ \\
\hline 4 & $0,12 \pm 0,01$ & $0,06 \pm 0,04$ & $0,11 \pm 0,01$ & $0,15 \pm 0,05$ & $0,06 \pm 0,06$ & $0,24 \pm 0,03$ \\
\hline 5 & $0,15 \pm 0,04$ & $0,08 \pm 0,02$ & $0,12 \pm 0,01$ & $0,15 \pm 0,05$ & $0,05 \pm 0,06$ & $0,19 \pm 0,04$ \\
\hline 6 & $0,10 \pm 0,03$ & $0,08 \pm 0,02$ & $0,11 \pm 0,03$ & $0,17 \pm 0,03$ & $0,06 \pm 0,04$ & $0,28 \pm 0,03$ \\
\hline 7 & $0,15 \pm 0,02$ & $0,09 \pm 0,03$ & $0,13 \pm 0,02$ & $0,15 \pm 0,01$ & $0,06 \pm 0,05$ & $0,16 \pm 0,02$ \\
\hline$\underline{8}$ & $0,12 \pm 0,01$ & $0,08 \pm 0,05$ & $0,09 \pm 0,03$ & $0,14 \pm 0,02$ & $0,06 \pm 0,08$ & $0,23 \pm 0,03$ \\
\hline
\end{tabular}

Resposta: absorbância da fase aquosa após 48 h nas condições desejadas, medida no comprimento de onda do máximo de absorção do corante

Tabela 6. Efeitos calculados a partir dos resultados obtidos com o planejamento fatorial $2^{3}$ para identificação da influência das condições sobre a solubilização de n-decano, tolueno e tetracloreto de carbono corados com Oil Blue e de tetracloreto de carbono corado com Oil Red por soluções $2,5 \%$ m/v de CTAB, SDS e Tween 40

\begin{tabular}{|c|c|c|c|c|c|c|c|c|c|c|}
\hline \multirow[b]{3}{*}{ EFEITOS } & \multicolumn{7}{|c|}{ Oil Blue } & \multicolumn{3}{|c|}{ Oil Red } \\
\hline & \multicolumn{2}{|c|}{ n-Decano } & \multicolumn{2}{|c|}{ Tolueno } & \multicolumn{3}{|c|}{ Tetracloreto de carbono } & \multicolumn{3}{|c|}{ Tetracloreto de carbono } \\
\hline & CTAB & SDS & СТAB & SDS & СТAB & SDS & Tween 40 & СТAB & SDS & Tween 40 \\
\hline Média & 0,255 & 0,176 & 0,359 & 0,200 & 0,135 & 0,078 & 0,114 & 0,186 & 0,106 & 0,200 \\
\hline 1 & 0,019 & 0,013 & 0,035 & 0,003 & $-0,048$ & $-0,008$ & $-0,003$ & 0,013 & 0,028 & 0,030 \\
\hline 2 & 0,002 & 0,010 & 0,01 & $-0,004$ & 0,014 & 0,011 & $-0,013$ & $-0,033$ & 0,008 & 0,120 \\
\hline 3 & $\mathbf{0 , 0 3 9}$ & $\mathbf{0 , 0 3 2}$ & 0,065 & 0,096 & $-0,004$ & 0,013 & 0,003 & $-0,023$ & 0,018 & $-0,035$ \\
\hline 12 & 0,021 & 0,002 & 0,007 & 0,019 & 0,004 & $-0,013$ & $-0,013$ & $-0,013$ & 0,013 & $-0,040$ \\
\hline 13 & 0,028 & 0,007 & $-0,012$ & 0,023 & 0,009 & $-8 \times 10^{-4}$ & $-0,008$ & $-0,023$ & $-0,018$ & $-0,005$ \\
\hline 23 & $-0,019$ & 0,007 & 0,007 & $-0,004$ & $-0,009$ & $-0,003$ & $-0,018$ & $-0,018$ & 0,013 & $-0,015$ \\
\hline 123 & $-8 \times 10^{-4}$ & 0,005 & $-0,003$ & 0,009 & 0,004 & 0,004 & 0,002 & 0,003 & 0,008 & 0,005 \\
\hline
\end{tabular}

respostas também não foram alteradas significativamente. Porém, quando se passa do nível inferior para o superior de temperatura (efeito 2) o efeito é significativo e positivo no caso da solução de Tween 40 e negativo para a solução de CTAB.

De acordo com os resultados obtidos no estudo da influência de cada um dos fatores isoladamente sobre o desempenho de solubilização, seria razoável esperar, por exemplo, que o efeito da temperatura fosse significativo e positivo na solubilização das NAPLs. Porém, o único efeito significativo e positivo da temperatura nos ensaios detectado com o planejamento fatorial realizado ocorreu na solubilização de tetracloreto de carbono corado com Oil Red pela solução de Tween 40. Além disto, os coeficientes de interação de $2^{\mathrm{a}}$ e $3^{\mathrm{a}}$ ordem não foram significativos, o que indica a possibilidade de que as modificações nas micelas dos surfactantes induzidas simultaneamente por diferentes condições de temperatura, $\mathrm{pH}$ e dureza sejam compensadas entre si, bem como ressalta a importância da realização de testes de seleção do surfactante mais indicado para a solubilização de uma determinada NAPL nas condições da fase aquosa esperadas na aplicação em campo.

\section{CONCLUSÕES}

Através de testes simples foi possível classificar o desempenho de soluções de surfactantes na solubilização de NAPLs. Verificou-se que cada solvente orgânico testado é mais bem solubilizado por um determinado surfactante e que a eficiência de solubilização é bastante influenciada pelo corante utilizado para a identificação da fase orgânica. Porém, os surfactantes não iônico Triton X-100 e catiônico CTAB foram os que apresentaram os melhores potenciais de aplicação na solubilização das NPLAs testadas. Para uma determinada NAPL e 
um mesmo corante, cada surfactante testado apresenta desempenhos diferentes quando temperatura, $\mathrm{pH}$ e dureza do meio aquoso são alteradas isoladamente. Os surfactantes que apresentam os melhores resultados de solubilização são também os que sofrem maiores influências quando as condições do meio são alteradas isoladamente. A utilização do planejamento fatorial permitiu detectar efeitos positivos na solubilização de tolueno e n-decano corados com Oil Blue para o surfactante SDS e, no caso do n-decano, para o surfactante CTAB, quando o pH aumenta. Já no caso do tetracloreto de carbono corado com Oil Red para o surfactante Tween 40, o efeito positivo surge quanto se aumenta a dureza. Para este mesmo par organoclorado/ corante com o surfactante CTAB foram detectados efeitos negativos quando se aumentam a temperatura e a dureza do meio.

Os testes realizados em escala de laboratório permitiram a identificação dos surfactantes com maior potencial de solubilização das NAPLs testadas e mostraram a importância da identificação da NAPL a ser solubilizada e dos parâmetros testados do meio $(\mathrm{pH}$, temperatura e dureza) na seleção adequada do surfactante a ser utilizado.

\section{MATERIAL SUPLEMENTAR}

No material suplementar, disponível em http://quimicanova.sbq. org.br, na forma de arquivo .PDF com acesso livre, encontram-se os gráficos referentes ao estudo com as DNAPLs, que ilustram a ocorrência da emulsificação de tetracloreto de carbono, a influência do corante no desempenho de solubilização de clorofórmio e 1,2-dicloroetano e, ainda, a influência de $\mathrm{pH}$, temperatura e dureza do meio sobre a solubilização de tetracloreto de carbono pelas soluções dos surfactantes testados.

\section{AGRADECIMENTOS}

À FAPESP (03/12529-4) e ao CNPq (310347/2006-8) pelo suporte financeiro e à PUC-Campinas pela disponibilização da infra-estrutura. À Profa. Dra. A. Borin pela leitura do texto e valiosas sugestões. As autoras M. R. Peres e S. B. de Moraes agradecem ao PIBIC/CNPq pelas bolsas de Iniciação Científica concedidas.

\section{REFERÊNCIAS}

1. Baird, C.; Química Ambiental, 2ª ed., Bookman: São Paulo, 2002.

2. http://www.vrom.nl/; http://www.umweltbundesamt.de/; http://www. fasp.info/; http://www.ovam.be/; http://www.umweltbundesamt.de/, acessadas em Agosto 2008.

3 http://www.cetesb.sp.gov.br/Solo/areas_contaminadas/texto_areas_cont_ nov_08.pdf, acessada em Agosto 2009.

4. Abdanur, A.; Dissertação de Mestrado, Universidade Federal do Paraná, Brasil, 2005.

5. Zhao, B.; Chen, X.; Zhu, K.; Colloids Surf., A 2007, 296, 167.

6. Geng, L.; Chen, Z.; Chan, C. W.; Huang, G. H.; Expert. Syst. Appl. 2001, $20,251$.

7. Ajo-Franklin, J. B.; Geller, T. J.; Harris, J. M.; Nguyen, T. T.; Youssef, N. H.; Mcinerney, M. J.; Sabatini, D. A.; Water Res. 2008, 42, 1735; Lee, Y-C.; Kwon, T.-S.; Yang, J.-S.; Yang, J.-W.; J. Hazard. Mater. 2007, 140, 340; Harwell, J. H.; Sabatini, D. A.; Knox, R. C.; Colloids Surf. A 1999, $151,255$.

8. Cassidy, N. J.; J. Contam. Hydrol. 2007, 94, 49; Kim, J. M.; Corapcioglu, Y.; J. Contam. Hydrol. 2003, 65, 137.
9. Moreira, C. A.; Aquino, W. F.; Dourado, J. C.; Rev. Bras. Geofís. 2007, $25,413$.

10. Dwarakanath, V.; Kostarelos, K; Pope, G. A.; Shotts, D.; Wade, W. H.; J. Contam. Hydrol. 1999, 38, 465; Yoon, H.; Valocchi, A. J.; Werth, C. J.; J. Contam. Hydrol. 2007, 90, 159.

11. Nobre, M. M.; Nobre, R. C. M.; Química e Derivados on line (2003), http://www.quimica.com.br/revista/qd417/solo2.htm, acessada em Fevereiro 2008; Moreira, C. A.; Dourado, J. C.; Rev. Bras. Geofis. 2005, $23,213$.

12. Flaming, J. E.; Knox, R. C.; Sabatini, D. A.; Kibbey, T. C.; Vadose Zone J. 2003, 2, 168; Zhao, B.; Zhu, L.; Yang, K.; Chemosphere 2006, 62, 772.

13. Mulligan, C. N.; Yong, R. N.; Gibbs, B. F.; Eng. Geol. 2001, 60, 371.

14. Schaerlaekens, J.; Feyen, J. ; J. Contam. Hydrol. 2004, 71, 283; Childs, J.; Acosta, E.; Annable, M. D. ; Brooks, M. C.; Enfield, C. G. ; Harwell, J. H.; Hasegawa, M.; Knox, R. C.; Rao, P. S.; Sabatini, D. A.; Shiau, B.; Szekeres, E.; Wood, A. L.; J. Contam. Hydrol. 2006, 82, 1; Barranco Jr, F. T.; Dawson, H. E.; Honeyman, B. D.; Environ. Sci. Technol. 1997, 31, 676; Qin, X. S.; Huang, G. H.; Chakma, A.; Water, Air, Soil Pollut. 2007, $185,349$.

15. Jeong, S-W.; Wood, A. L.; Lee, T. R.; J. Hazard. Mater. 2002, 95, 125.

16. Lide, D. R., ed.; CRC Handbook of Chemistry and Physics, $84^{\text {th }}$ ed., CRC Press: Boca Raton, 2004-2005.

17. Timm, L. C.; Pires, L. F.; Roveratti, R.; Arthur, R. C. J.; Reichardt, K.; Oliveira, J. C. M.; Bacchi, O. O. S.; Sci. Agric. (Piracicaba, Braz.) 2006, 63, 55; Běter-Rogač, M.; Acta Chim. Slov. 2007, 54, 452; Acharya, K. R.; Bhattacharyya, S. C.; Moulik, S. P.; J. Photochem. Photobiol. A 1999, 122, 47.

18. Zhou, M.; Rhue, R. D.; Environ. Sci. Technol. 2000, 34, 1985.

19. Imazaki, L. T.; Coelho, L. H. S.; Peres, M. R.; Souza, E. F.; Anais do XIV Congresso Brasileiro de Águas Subterrâneas, Curitiba, Brasil, 2006.

20. Teófilo, R. F.; Ferreira, M. M. C.; Quim. Nova 2006, 29, 338; http://lqta. iqm.unicamp.br/ acessada em Agosto 2008.

21. Shaw, D. J.; Introduction to colloid and surface chemistry, $4^{\text {th }}$ ed., Butterworth-Heinemann: Oxford, 2003.

22. Choi, T.-S.; Shimizu, Y.; Shirai, H.; Hamada, K.; Dyes Pigm. 2000, 45, 145.

23. Gutiérrez, C.; Ziegler, G.; Trötschler, O.; Braun J.; J. Contamin. Hydrol. 2003, 67, 61 .

24. Roy, J. W.; Smith, J. E.; Gillham, R. W.; J. Contamin. Hydrol. 2004, 74, 145; Jeong, S.-W.; J. Colloid Interface Sci. 2005, 282, 182.

25. van Valkenburgn, M. E.; Annable, M. D.; J. Contamin. Hydrol. 2002, 59 , 211.

26. Neogi, P.; Munden, J. J.; J. Colloid Interface Sci. 2007, 305, 202.

27. Cui, Z.; Canselier, J. P.; Polym. Int. 2003, 52, 548.

28. Bloor, J. R.; Morrison, J. C.; Rhodes, C. T.; J. Pharm. Sci. 1969, 59, 387.

29. Amaral, A. S.; Anghinoni, I.; Pesq. Agropec. Bras. 2001, 36, 695.

30. Mitra, R. M.; Sinha, S. S.; Pal, S. K.; J. Phys. Chem. B 2007, 111, 7577; Liu, Y.; Chen, S.-H.; Huang, J. S.; Macromolecules 1998, 31, 2236.

31. Kooreman, P. A.; Engberts, J. B. F. N.; van Os, N. M.; J. Surfactants Deterg. 1998, 1, 23.

32. Imhoff, P. T.; Frizzel, A.; Miller, C. T.; Environ. Sci. Technol. 1997, 31, 1615.

33. Abed, M. A.; Saxena, A.; Bohidar, H. B.; Colloids Surf., A 2004, 233, 181.

34. Stoner, M. R.; Dale, D. A.; Gualfetti, P. J.; Bechker, T.; Randolph, T. W.; Biotechnol. Prog. 2005, 21, 1716. 


\section{AVALIAÇÃO DO DESEMPENHO DE SURFACTANTES PARA A SOLUBILIZAÇÃO DE FASES LÍQUIDAS NÃO AQUOSAS EM MEIO AQUOSO}

Elizabeth Fátima de Souza*, Mariana Rodrigues Peres e Samara Boaventura de Moraes

Faculdade de Química, Centro de Ciências Exatas, Ambientais e de Tecnologias, Pontifícia Universidade Católica de Campinas, CP 317, 13012-970 Campinas - SP, Brasil

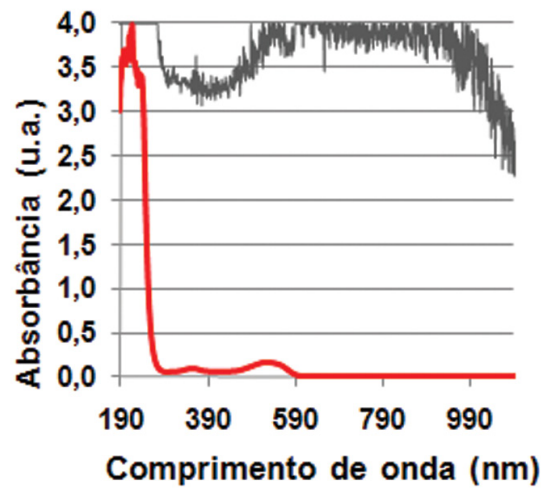

Figura 1S. Espectros UV/vis da fase aquosa de soluções de surfactantes 2,5\% $\mathrm{m} / \mathrm{v}$ contaminadas com tetracloreto de carbono corado com Oil Red, após 48 $h$ de contato na temperatura de $25^{\circ} \mathrm{C}$

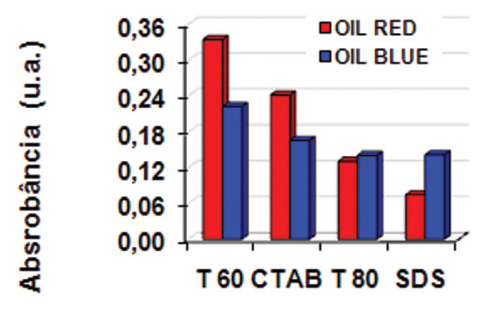

a)

Surfactante

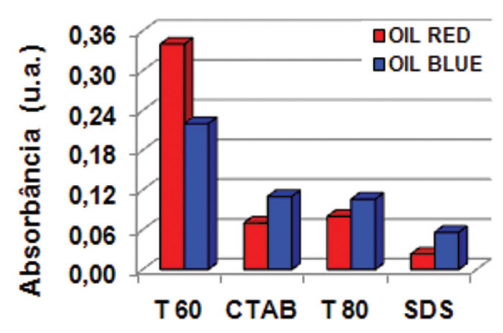

b)

\section{Surfactante}

Figura 2S. Comparação do desempenho de solubilização de clorofórmio (a) e 1,2-dicloroetano (b) corados com Oil Red ou Oil Blue por soluções $2,5 \% \mathrm{~m} / \mathrm{v}$ dos surfactantes CTAB, SDS, Tween 60 ou Tween 80 , após $48 \mathrm{~h}$ de contato na temperatura de $25^{\circ} \mathrm{C}$, por medida da absorbância da fase aquosa no comprimento de onda típico do corante
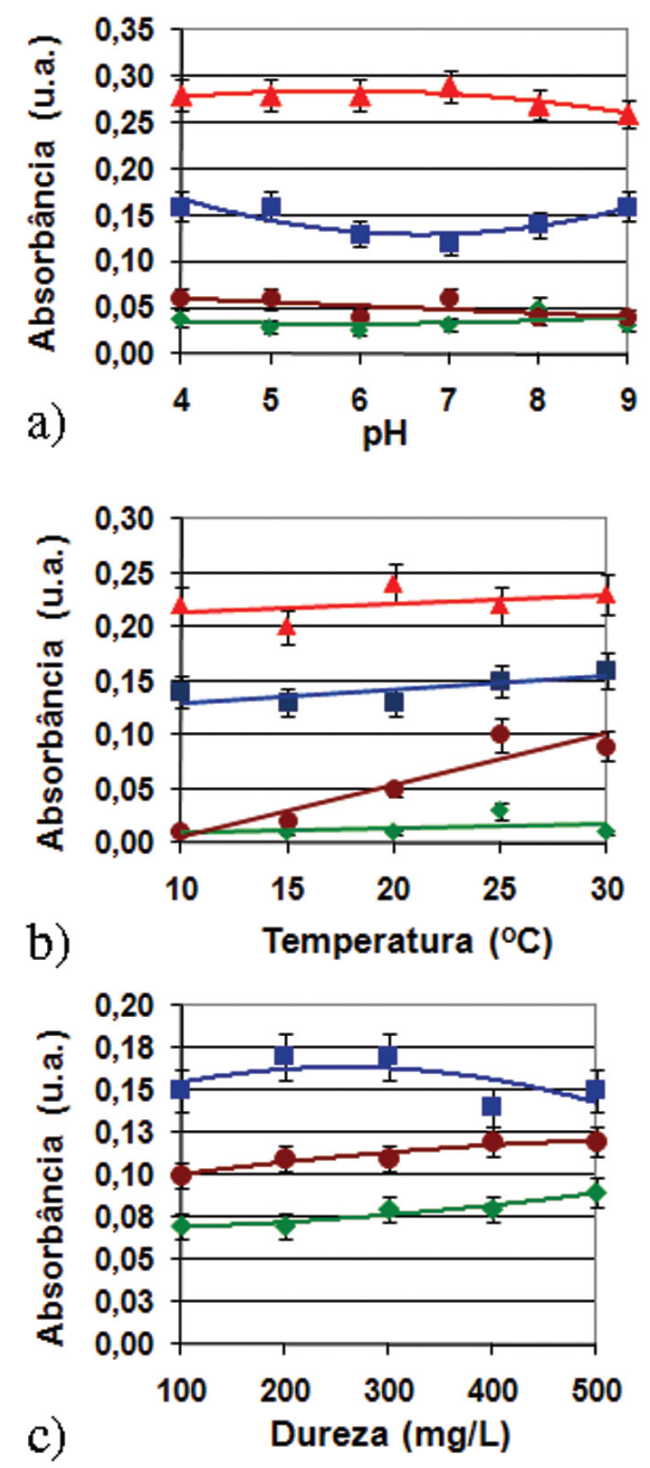

Figura 3S. Efeito da temperatura (a), do pH (b) e da dureza (c) na solubilização de tetracloreto de carbono corado com Oil Red por soluções 2,5\% $\mathrm{m} / \mathrm{v}$ dos surfactantes CTAB, SDS, Tween 80 ou Triton X100, após 48 h de contato na temperatura indicada ou a $25^{\circ} \mathrm{C}$ ( pH e dureza), por medida da absorbância da fase aquosa no comprimento de onda típico do corante 